\title{
Free Radical Copolymerization: An NMR Investigation of Current Kinetic Models
}

\author{
Ian A. Maxwell, Annemieke M. Aerdts, and Anton L. German \\ Department of Polymer Chemistry and Technology, University of Technology, \\ 5600 MB Eindhoven, The Netherlands
}

Received June 4, 1992; Revised Manuscript Received November 23, 1992

\begin{abstract}
The ability of terminal model kinetics to predict copolymer microstructure was tested for the styrene-methyl methacrylate system copolymerized at $40^{\circ} \mathrm{C}$ at various fractions of monomers. Carbon- 13 NMR studies (in the accompanying paper) showed that previous peak assignments of the spectra of statistical styrene-methyl methacrylate copolymers were incorrect. The peak areas obtained for all copolymers were described in terms of triad sequences and were also adequately predicted by the terminal model utilizing reactivity ratios calculated from a nonlinear least squares fit to the copolymer composition data determined by ${ }^{1} \mathrm{H}$ NMR. The nonterminal model behavior of the kinetics of the rate of polymerization described elsewhere can be successfully modeled by the "restricted" penultimate model of Fukuda et al. We show that all current data can also be explained by various "bootstrap" or monomer-polymer complex models. Further model discrimination awaits new independent experimental results.
\end{abstract}

\section{Introduction}

The kinetic model that is most widely used to model free radical copolymerizations is the terminal model, also known as the Mayo-Lewis model. ${ }^{1}$ For most copolymer systems the copolymer composition has been shown to be well fitted by the terminal model equation. ${ }^{2}$ Recently, however, there have been numerous reports of the inability of the terminal model to fit other types of copolymerization data. ${ }^{3-7}$ These discrepancies have been explained in at least two ways, which are described below.

First, the rate or mean propagation rate coefficient of copolymerization has been found not to obey the terminal model.., 6 This deviation has been explained by invoking the penultimate model for copolymerization, in which the penultimate group from the polymeric free radical end is considered to affect the reactivity of the radical. In order to accommodate the fact that composition is well fitted by the terminal model, a restricted penultimate model has been developed ${ }^{5,8}$ in which the terminal model is a limit for the case of polymer composition.

Copolymer composition and monomer sequence distribution data for some copolymers have been shown to be sensitive to the solvent utilized during polymerization. ${ }^{7,9}$ A model which has been invoked to explain the effect of solvents upon both sequence distributions and copolymer composition in copolymerization is the so-called "bootstrap" model. ${ }^{7}$ This model was derived from the observation that copolymers of the same composition have the same microstructure irrespective of the solvent used during copolymerization. The assumption is that the conditional probabilities governing the propagation reactions are independent of the solvent. This model has been successful in explaining solvent effects in copolymerization.

One of the current difficulties in the field of free radical copolymerization is discrimination between models (there are at least 10 models for copolymerization $n^{1,3,7,10}$ ). On the basis of composition and rate data O'Driscoll and Davis ${ }^{11}$ stated that nuclear magnetic resonance (NMR) experiments are incapable of model discrimination. However, Moad et al. ${ }^{12}$ showed that this argument assumed that an adequate fit to polymer composition data of the terminal

* To whom correspondence should be addressed. Current address: Sydney University Polymer Centre, School of Chemistry, University of Sydney, NSW 2006, Australia. model justified the use of the terminal model. In fact, Moad et al. ${ }^{12}$ showed that an adequate fit to composition data by the terminal model does not discount penultimate unit effects on polymer composition. Further, these authors ${ }^{12}$ pointed out the necessity of further experimental information before model discrimination in copolymerization could proceed. This is the aim of this paper.

Many workers have assumed that the inadequacy of the terminal model in some cases automatically suggests penultimate unit effects, despite the fact that numerous other models of copolymerization have sufficient adjustable parameters to fit all current data. In fact, to our knowledge there is only one claimed experimental (electron spin resonance (ESR)) observation of a penultimate unit effect, ${ }^{13}$ and that is of the rather exotic homopolymerization (or dimerization?) of tert-butyl $\alpha$-tert-butylsulfenyl acrylate with various azo initiators in chlorobenzene at 24 ${ }^{\circ} \mathrm{C}$. The substituent groups on the initiator fragment were claimed to affect the ESR signal of the propagating radical. It is unclear why this would be the case following a few propagation steps after which substituents on the initiator would be unlikely to affect the propagating radical (obviously an oligomeric radical with degree of polymerization (dp) $>2$ cannot feel penultimate unit effects from the initiator fragment, simply because the penultimate unit is not the initiator fragment). Without further experimental details it is unlikely that these experimental data can be used as evidence for penultimate unit effects in bulk copolymerization.

In this paper we endeavor to test a limited number of models for copolymerization by measuring the ${ }^{13} \mathrm{C}$ NMR triad sequence distributions for the "model" bulk copolymerization system styrene and methyl methacrylate (MMA) at $40^{\circ} \mathrm{C}$. These data may allow the discrimination between both terminal and penultimate unit effects and any other effects on copolymer composition data. Previous attempts to fit triad sequence distributions of this system by the terminal model are invalid since they utilize ${ }^{1} \mathrm{H}$ NMR spectra, which have been shown to be due to (at least) pentad sequences. ${ }^{14}$ Further, in the accompanying paper ${ }^{15}$ we show that all previous triad sequence assignments of ${ }^{13} \mathrm{C}$ NMR spectra are invalid and justify new assignments for these spectra. One further advantage of ${ }^{13} \mathrm{C}$ NMR is that both styrene-centered and MMA-centered triad sequence distributions can be measured. ${ }^{15}$ The ${ }^{13} \mathrm{C}$ NMR sequence distributions are measured for polymers 
polymerized at conditions utilized by Fukuda et al..$^{5}$ to determine the mean propagation rate coefficients for this system. We also determine the ${ }^{1} \mathrm{H}$ NMR polymer composition data, and with the mean propagation rate coefficient and triad sequence distribution data this provides the most complete set of copolymerization data on this system to date.

\section{Theory}

Terminal Model. The terminal model for copolymerization assumes that there are two types of polymeric radicals distinguished by their end units, $\mathrm{S}^{\circ}$ and $\mathrm{M}^{\circ}$, where $\mathrm{S}$ and $\mathrm{M}$ represent the two monomer units in the system (in this study these represent styrene and MMA, respectively). The rate of copolymerization is given by the rate of decrease of the total monomer concentration, $C_{\mathrm{m}}$.

$$
-\frac{\mathrm{d} C_{\mathrm{m}}}{\mathrm{d} t}=-\frac{\mathrm{d}[\mathrm{S}]}{\mathrm{d} t}-\frac{\mathrm{d}[\mathrm{M}]}{\mathrm{d} t}=k_{\mathrm{p}}([\mathrm{S}]+[\mathrm{M}])\left(\left[\mathrm{S}^{\circ}\right]+\left[\mathrm{M}^{\circ}\right]\right)
$$

where $\left[\mathrm{S}^{\circ}\right]$ and $\left[\mathrm{M}^{\circ}\right]$ are the concentrations of the polymeric free radicals, $[\mathrm{S}]$ and $[\mathrm{M}]$ are the concentrations of the monomers, and $k_{\mathrm{p}}$ is the mean propagation rate coefficient given $b^{5}$

$$
k_{\mathrm{p}}=\frac{r_{\mathrm{s}} f_{\mathrm{s}}^{2}+2 f_{\mathrm{g}} f_{\mathrm{m}}+r_{\mathrm{m}} f_{\mathrm{m}}^{2}}{r_{\mathrm{s}} f_{\mathrm{s}} / k_{\mathrm{ss}}+r_{\mathrm{m}} f_{\mathrm{m}} / k_{\mathrm{mm}}}
$$

where $f_{\mathrm{s}}$ and $f_{\mathrm{m}}$ are the mole fractions of each monomer in the reaction and $r_{\mathrm{s}}$ and $r_{\mathrm{m}}$ are the reactivity ratios given by

$$
r_{\mathrm{s}}=k_{\mathrm{sg}} / k_{\mathrm{sm}} \quad r_{\mathrm{m}}=k_{\mathrm{mm}} / k_{\mathrm{ms}}
$$

where $k_{\mathrm{ij}}$ is the propagation rate coefficient for radical I ${ }^{\circ}$ with monomer $\mathrm{J}(\mathrm{i}, \mathrm{j}=\mathrm{s}$ or $\mathrm{m} ; \mathrm{I}, \mathrm{J}=\mathrm{S}$ or $\mathrm{M})$. The instantaneous copolymer composition is given by the wellknown copolymer equation ${ }^{1}$

$$
\frac{F_{\mathrm{s}}}{F_{\mathrm{m}}}=\frac{f_{\mathrm{s}}\left(r_{\mathrm{s}} f_{\mathrm{s}}+f_{\mathrm{m}}\right)}{f_{\mathrm{m}}\left(r_{\mathrm{m}} f_{\mathrm{m}}+f_{\mathrm{s}}\right)}
$$

where $F_{\mathrm{s}}$ and $F_{\mathrm{m}}$ are the mole fractions of monomers $\mathrm{S}$ and $M$ in the copolymer.

In this paper we measure the ${ }^{13} \mathrm{C}$ NMR spectra, of which some of the resonances can be assigned in terms of triad sequence distributions. These are given in terms of conditional probabilities, which themselves can be couched in terms of monomer ratios and reactivity ratios. We define the quantity $x$ as the ratio of the monomer concentrations:

$$
x=[\mathrm{S}] /[\mathrm{M}]
$$

The fractions of each type of styrene-centered triad are given by ${ }^{16}$

$$
\begin{gathered}
F_{\mathrm{sss}}=\frac{r_{\mathrm{s}}{ }^{2} x^{2}}{1+2 r_{\mathrm{s}} x+r_{\mathrm{s}}{ }^{2} x^{2}} \\
F_{\mathrm{msm}}=\frac{1}{1+2 r_{\mathrm{s}} x+r_{\mathrm{s}}{ }^{2} x^{2}} \\
F_{\mathrm{mss}}=F_{\mathrm{ssm}}=\frac{r_{\mathrm{s}} x}{1+2 r_{\mathrm{s}} x+r_{\mathrm{s}}{ }^{2} x^{2}}
\end{gathered}
$$

Similarly, the fractions of each type of MMA-centered triads are given by ${ }^{16}$

$$
F_{\mathrm{mmm}}=\frac{r_{\mathrm{m}}{ }^{2} / x^{2}}{1+2 r_{\mathrm{m}} / x+r_{\mathrm{m}}{ }^{2} / x^{2}}
$$

$$
\begin{gathered}
F_{\mathrm{sms}}=\frac{1}{1+2 r_{\mathrm{m}} / x+r_{\mathrm{m}}{ }^{2} / x^{2}} \\
F_{\mathrm{mms}}=F_{\mathrm{smm}}=\frac{r_{\mathrm{m}} / x}{1+2 r_{\mathrm{m}} / x+r_{\mathrm{m}}{ }^{2} / x^{2}}
\end{gathered}
$$

Penultimate Model. The penultimate model considers that there are four distinct types of polymeric free radicals and that each is distinguished by the terminal and the penultimate monomer units from the radical site. In the penultimate model the mean propagation rate coefficient is given by ${ }^{5}$

$$
k_{\mathrm{p}}=\frac{F_{\mathrm{s}_{\mathrm{s}}}{ }^{2}+2 f_{\mathrm{s}} f_{\mathrm{m}}+\bar{r}_{\mathrm{m}} f_{\mathrm{m}}^{2}}{F_{\mathrm{s}} / \bar{k}_{\mathrm{ss}}+\bar{F}_{\mathrm{m}} f_{\mathrm{m}} / \bar{k}_{\mathrm{mm}}}
$$

where the mean reactivity ratios and propagation rate coefficients are given by

$$
\begin{gathered}
F_{\mathrm{s}}=\frac{r_{\mathrm{s}}^{\prime}\left[f_{\mathrm{s}} r_{\mathrm{s}}+f_{\mathrm{m}}\right]}{f_{\mathrm{s}} r_{\mathrm{s}}^{\prime}+f_{\mathrm{m}}} \\
F_{\mathrm{m}}=\frac{r_{\mathrm{m}}\left[f_{\mathrm{m}} r_{\mathrm{m}}+f_{\mathrm{s}}\right]}{f_{\mathrm{m}} r_{\mathrm{m}}{ }^{\prime}+f_{\mathrm{s}}} \\
k_{\mathrm{ss}}=\frac{k_{\mathrm{sss}}\left[f_{\mathrm{s}} r_{\mathrm{s}}+f_{\mathrm{b}}\right]}{f_{\mathrm{s}} r_{\mathrm{s}}+f_{\mathrm{m}} / s_{\mathrm{s}}} \\
k_{\mathrm{mm}}=\frac{k_{\mathrm{mmm}}\left[f_{\mathrm{m}} r_{\mathrm{m}}+f_{\mathrm{s}}\right]}{f_{\mathrm{m}} r_{\mathrm{m}}+f_{\mathrm{s}} / s_{\mathrm{m}}}
\end{gathered}
$$

where the reactivity ratios are

$$
\begin{array}{ll}
r_{\mathrm{i}}=k_{\mathrm{iii}} / k_{\mathrm{ijj}} & \mathrm{i}, \mathrm{j}=\text { s or } \mathrm{m}, \mathrm{i} \neq \mathrm{j} \\
r_{\mathrm{i}}^{\prime}=k_{\mathrm{jii}} / k_{\mathrm{jij}} & \mathrm{i}, \mathrm{j}=\mathrm{s} \text { or } \mathrm{m}, \mathrm{i} \neq \mathrm{j} \\
s_{\mathrm{i}}=k_{\mathrm{jii}} / k_{\mathrm{iii}} & \mathrm{i}, \mathrm{j}=\mathrm{s} \text { or } \mathrm{m}, \mathrm{i} \neq \mathrm{j}
\end{array}
$$

where the propagation rate coefficients for addition of monomer $\mathrm{J}$ to radical $\mathrm{II}^{\circ}$ is given by $k_{\mathrm{iij}}(\mathrm{i}, \mathrm{j}=\mathrm{s}$ or $\mathrm{m} ; \mathrm{I}, \mathrm{J}$ = $\mathrm{S}$ or $\mathrm{M}$ ).

The instantaneous copolymer composition is given by the penultimate copolymer equation ${ }^{5}$

$$
\frac{F_{\mathrm{s}}}{F_{\mathrm{m}}}=\frac{f_{\mathrm{s}}\left(\bar{F}_{\mathrm{s}} f_{\mathrm{s}}+f_{\mathrm{m}}\right)}{f_{\mathrm{m}}\left(\bar{r}_{\mathrm{m}} f_{\mathrm{m}}+f_{\mathrm{s}}\right)}
$$

The fractions for the styrene-centered triads are ${ }^{16}$

$$
\begin{gathered}
F_{\mathrm{sss}}=\frac{r_{\mathrm{s}} r_{\mathrm{s}} x^{2}}{1+2 r_{\mathrm{s}}{ }^{\prime} x+r_{\mathrm{s}} r_{\mathrm{s}}^{\prime} x^{2}} \\
F_{\mathrm{msm}}=\frac{1}{1+2 r_{\mathrm{s}}^{\prime} x+r_{\mathrm{s}} r_{\mathrm{s}}{ }^{\prime} x^{2}} \\
F_{\mathrm{ssm}}=F_{\mathrm{mss}}=\frac{r_{\mathrm{s}}^{\prime} x}{1+2 r_{\mathrm{s}}^{\prime} x+r_{\mathrm{s}} r_{\mathrm{s}}^{\prime} x^{2}}
\end{gathered}
$$

Similarly, for the MMA-centered triads ${ }^{16}$

$$
\begin{gathered}
F_{\mathrm{mmm}}=\frac{r_{\mathrm{m}} r_{\mathrm{m}}^{\prime} / x^{2}}{1+2 r_{\mathrm{m}}^{\prime} / x+r_{\mathrm{m}} r_{\mathrm{m}}^{\prime} / x^{2}} \\
F_{\mathrm{sms}}=\frac{1}{1+2 r_{\mathrm{m}}^{\prime} / x+r_{\mathrm{m}} r_{\mathrm{m}}^{\prime} / x^{2}} \\
F_{\mathrm{smm}}=F_{\mathrm{mms}}=\frac{r_{\mathrm{m}}^{\prime} / x}{1+2 r_{\mathrm{m}}^{\prime} / x+r_{\mathrm{m}} r_{\mathrm{m}}^{\prime} / x^{2}}
\end{gathered}
$$

The Bootstrap Model. Recently, the bootstrap model for copoolymerization has been developed ${ }^{7}$ for which the 


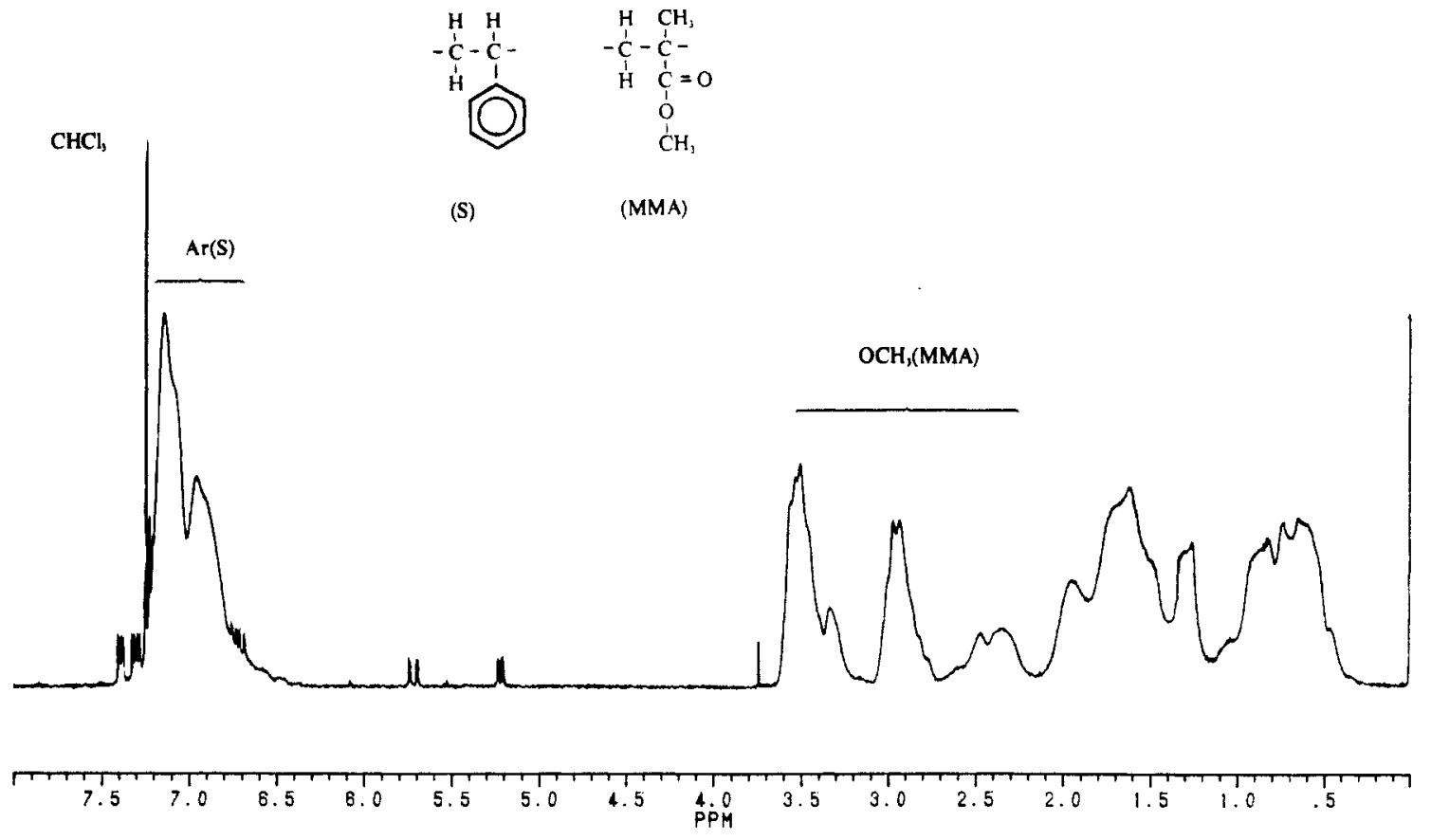

Figure 1. $400-\mathrm{MHz}{ }^{\mathrm{H}} \mathrm{H}$ NMR spectrum of a low-conversion bulk styrene-MMA copolymer (fraction of styrene in feed, $f_{\mathrm{s}}=0.377$ ) in $\mathrm{CDCl}_{3}$ at $298 \mathrm{~K}$. Styrene is represented by $\mathrm{S}$. The symbol $\mathrm{Ar}(\mathrm{S})$ represents the resonance of the aromatic protons on the styrene unit, and $\mathrm{OCH}_{3}(\mathrm{MMA})$ represents the resonance of the methoxy protons on the MMA unit.

primary assumption is that the monomer ratio at the polymerization site is different from that in the bulk. Further, the monomer ratio is said to have no effect upon the conditional probabilities or reactivity ratios. Therefore polymers of the same overall composition in different solvents always have the same microstructure.

In principle, the bootstrap model considers the actual or true monomer concentrations at the polymerization site, $\left[S_{t}\right]$ and $\left[\mathrm{M}_{\mathrm{t}}\right]$, and allows the ratio of these quantities to be related to the ratio of the bulk monomer concentrations by an equilibrium constant, $K$. It has been suggested 7 that the true ratio of the monomers at the polymerization site, $x_{t}$, is given by

$$
x_{\mathrm{t}}=\frac{\left[\mathrm{S}_{\mathrm{t}}\right]}{\left[\mathrm{M}_{\mathrm{t}}\right]}=K \frac{[\mathrm{S}]}{[\mathrm{M}]}
$$

Note that this equation assumes that the bootstrap effect is independent of factors such as monomer ratio and polymer composition; i.e., a single coefficient, $K$, describes the bootstrap effect. In principle, this equation can be applied to any other model for copolymerization. That is all quantities in, say, the terminal model that relate to the monomer concentrations, i.e., $f_{\mathrm{s}}, f_{\mathrm{m}}$, and $x$, must be replaced by their true value as defined by the equilibrium constant, $K$.

\section{Experimental Section}

The experimental details are given briefly in this paper and are discussed in detail in the accompanying paper. ${ }^{15}$ The rationalization for separating the work into two papers is that careful study of the ${ }^{13} \mathrm{C}$ NMR spectra established new peak area assignments for statistical styrene-MMA copolymers. This in itself is a significant result, independent of the application of the data to this kinetic study.

Materials. Styrene and MMA (Merck) were distilled at reduced pressure under nitrogen. The middle fraction of the distillate was collected and used. The initiator, $2,2^{\prime}$-azobis(isobutyronitrile) (AIBN) p.a. (Fluka) was recrystallized once from methanol.

Bulk Polymerizations. The copolymers were synthesized in bulk at $40^{\circ} \mathrm{C}$ in a $100-\mathrm{mL}$ glass vessel. Prior to polymerization the monomer solution was degassed, and the polymerization was performed under a $\mathrm{N}_{2}$ atmosphere. The initiator (AIBN) concentration was $2 \times 10^{-2} \mathrm{~mol} \mathrm{dm}^{-3}$. Final conversion was measured by gravimetry and was always less than $3 \%$. Isolation of the polymer was performed by pouring the final reaction mixture into a 10 -fold excess of cold heptane. The decanted polymers were dried in a vacuum oven at $50^{\circ} \mathrm{C}$.

NMR. ${ }^{1} \mathrm{H}$ NMR spectra were recorded with a $400-\mathrm{MHz}$ spectrometer (Bruker AM 400) at $298 \mathrm{~K}$, using $\mathrm{CDCl}_{3}$ as solvent and locking agent. A typical ${ }^{1} \mathrm{H}$ NMR spectrum is displayed in Figure 1. ${ }^{13} \mathrm{C}$ NMR spectra were recorded at $100 \mathrm{MHz}$ (Bruker AM 400 spectrometer) at $298 \mathrm{~K}$, using a sample concentration $10 \%(\mathrm{w} / \mathrm{v})$ in $\mathrm{CDCl}_{3}$. A typical ${ }^{13} \mathrm{C} \mathrm{NMR}$ spectrum is displayed in Figure 2. Peak areas were determined with a planimeter. The precision in the measurement of the peak areas was estimated to be within $5 \%$ (from a consideration of normal errors associated with quantitative NMR). Further details of the NMR techniques are given in the accompanying paper. ${ }^{15}$

In performing quantitative NMR measurements via compositional or configurational sequence placement, one must take into account differences in nuclear Overhauser effects (NOE) and spin-lattice relaxation times $\left(T_{1}\right)$. No NOE or $T_{1}$ values were initially determined in this work, but an additional ${ }^{13} \mathrm{C}$ NMR experiment was performed on one sample with a much longer delay $(15 \mathrm{~s})$ and gating off the decoupler to remove the NOE. The results were identical with those obtained via ${ }^{13} \mathrm{C}$ NMR with the standard method. We assume that no differential $T_{1}$ values are present for different stereoisomeric triads or compositional triads in the ${ }^{13} \mathrm{C}$ NMR spectra. Within these limits relative peak areas are proportional to the number of carbon atoms involved.

\section{Results and Discussion}

Testing of Models. In this work we have used a nonlinear least squares fitting technique known as the functional relationship model ${ }^{17}$ which allows the fitting of multiparameter models to data. One important feature of this technique is that it encompasses, as an input parameter, an estimate of the random error (precision) in both the experimentally observed dependent and independent variables (estimated at $5 \%$ for the peak areas and $1 \%$ for the fraction of monomer in the feed). The errors indicated for all fitted parameters are standard deviations which can be related to both the variance and confidence limits through the variance-covariance ma- 

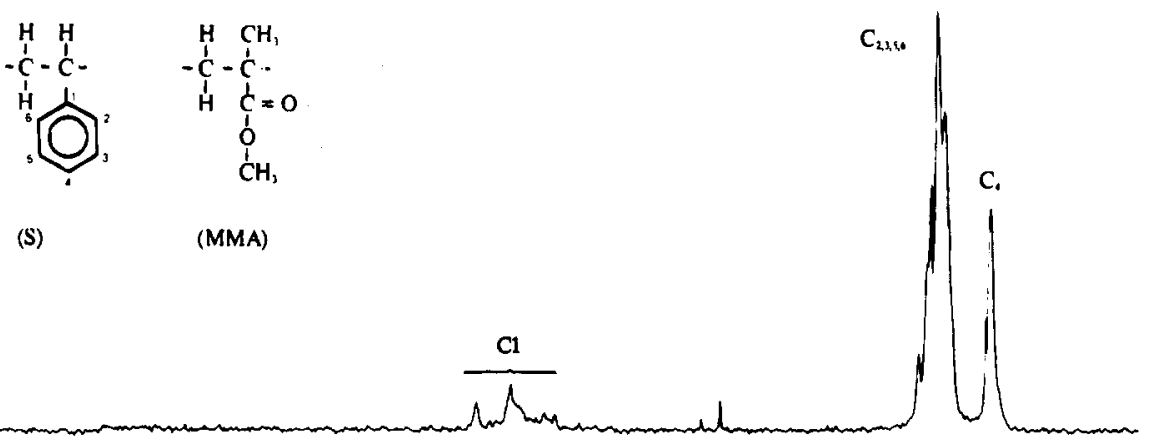

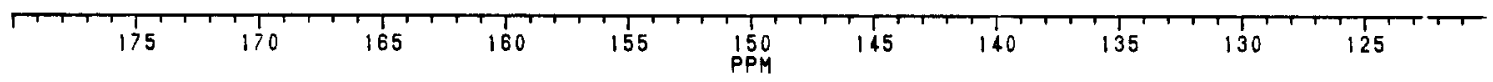

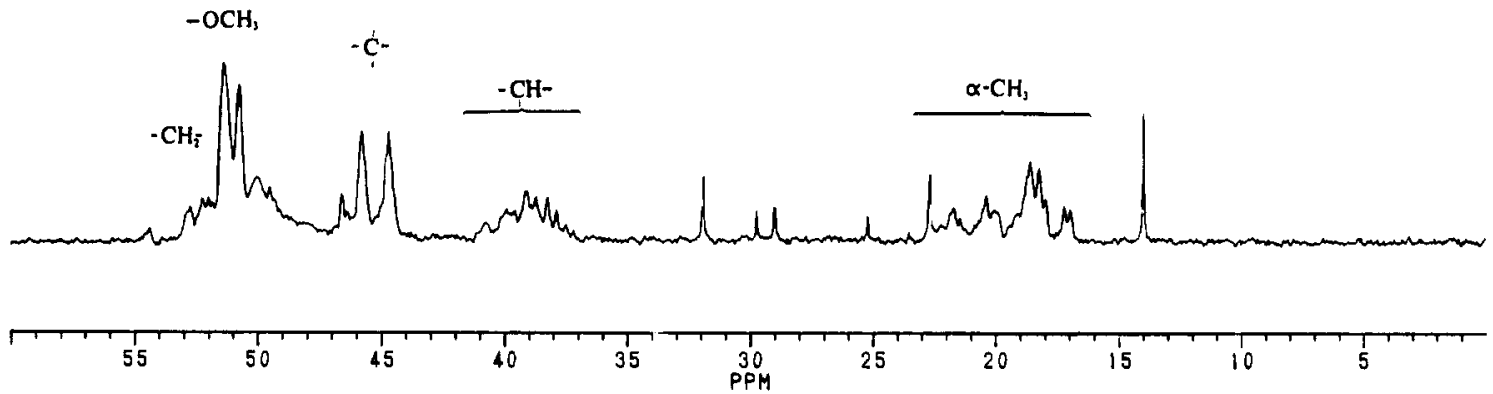

Figure 2. $100-\mathrm{MHz}^{13} \mathrm{C}$ NMR spectrum of a low-conversion bulk styrene-MMA copolymer (fraction of styrene in feed, $f_{8}=0.377$ ) in $\mathrm{CDCl}_{3}$ at $298 \mathrm{~K}$. Styrene is represented by $\mathrm{S}$. $\mathrm{C}=0$ represents the resonance of the carbonyl carbon on the MMA unit, $\mathrm{C} x$ (where $x=1-6$ ) represents the aromatic carbon resonances on the styrene unit, $\mathrm{CH}_{2}$ represents the main-chain carbon resonances, $\mathrm{OCH}_{3}$ represents the methoxy carbon resonances on the MMA unit, $\mathrm{C}$ represents the main-chain carbon resonance on the MMA unit, $\mathrm{CH}$ represents the main-chain carbon resonance on the styrene unit, and $\alpha-\mathrm{CH}_{3}$ represents the $\alpha$-methyl resonance on the MMA unit.

trix. ${ }^{17}$ If the fit of the model to data is valid, the size of the standard deviation indicates the significance of the fit. Alternatively, if there is more than one fitted parameter (which is not the case for the fit of the terminal model to ${ }^{13} \mathrm{C}$ NMR peak areas), joint confidence regions can be constructed, although in this work this has not been done, simply because of the number and complexity of models discussed.

Copolymer Composition. The motivation for utilizing copolymerization conditions exactly the same as that used by Fukuda et al. ${ }^{5}$ was to obtain copolymer triad sequence distribution data and hence obtain a full set of copolymerization data, namely, the mean propagation rate coefficients, the copolymer composition, and the triad sequence distributions, all at various monomer ratios. Hence, before further analysis of the triad sequence data was performed, we confirmed that the copolymer compositions of the bulk polymers measured by ${ }^{1} \mathrm{H}$ NMR were similar to those measured by Fukuda et al. In Table I these data are presented, and in Figure 3 the copolymer compositions from the two sets of experiments are presented.

The terminal model (eq 4) was fitted to the polymer composition data of both Fukuda et al. ${ }^{5}$ and this work. For the polymer compositions measured by Fukuda et al. we found the following reactivity ratios: $r_{s}=0.53 \pm 0.02$ and $r_{m}=0.49 \pm 0.02$, where the subscripts $s$ and $m$ refer to styrene and MMA, respectively. These values are similar to those fitted by Fukuda et al., $r_{\mathrm{s}}=0.523$ and $r_{\mathrm{m}}$ $=0.460$, for the same data. The composition data from this work were fitted by the same technique as above and gave $r_{s}=0.48 \supset 0.03$ and $r_{m}=0.42 \pm 0.09$. These values agree well with values proposed by $O^{\prime}$ Driscoll et al. ${ }^{18}$ for the copolymerization of styrene and methyl methacrylate, namely, $r_{\mathrm{s}}=0.472$ and $r_{\mathrm{m}}=0.454$. Therefore the composition data of this study suggest that the copolymers
Table I

Comparison of Copolymer Compositions from Bulk Polymerizations Performed in This Work and by Fukuda et al. ${ }^{5}$ as Well as the Final Conversions of Copolymerizations Performed in This Work and the Mean Propagation Rate Coefficient Measured by Fukuda et al. ${ }^{3}$

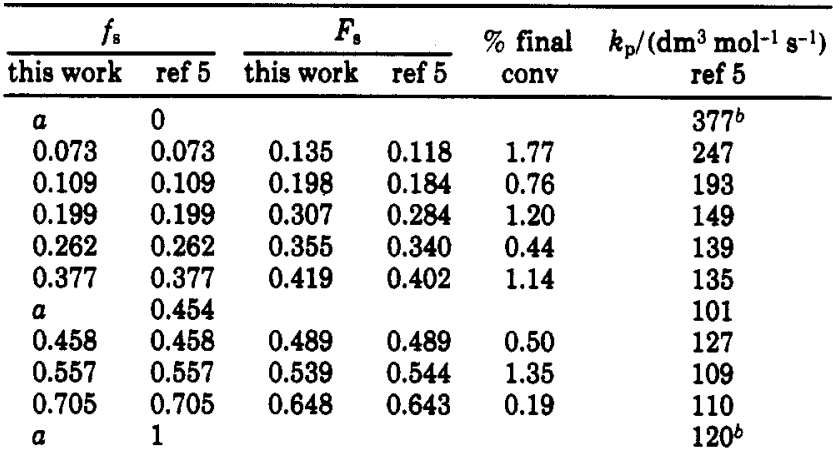

${ }^{a}$ Not polymerized in this work. ${ }^{b}$ Average of values in ref 5 .

studied in this work are those normally produced by these polymerizations.

Triad Sequence Distributions. For statistical copolymers there are 20 possible configurations of triads, 10 for each of the styrene-centered and MMA-centered triads. The fraction of each triad is given by $F_{i i i}$, where $\mathrm{i}=\mathrm{s}$ or $m$. The 20 possible configurations in terms of triad fractions are given in Tables II and III. The probability that two adjacent monomer units have a meso configuration (isotactic) is given by a tacticity parameter $\sigma$. The coisotacticity parameter, $\sigma_{\mathrm{sm}}$, is calculated ${ }^{15}$ from the methoxy region in the ${ }^{1} \mathrm{H}$ NMR spectra and is 0.44 . The other tacticities, $\sigma_{\mathrm{ss}}$ and $\sigma_{\mathrm{mm}}$, are taken from ${ }^{13} \mathrm{C}$ spectra of the homopolymers, and are 0.29 and 0.23 , respectively. ${ }^{15}$ 


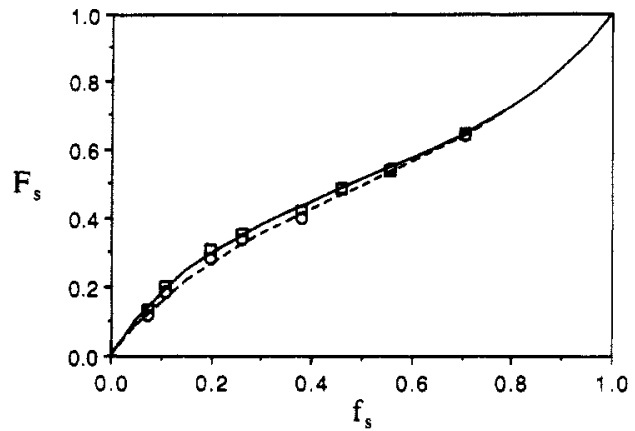

Figure 3. Fraction of styrene in copolymer versus fraction of styrene in feed. Experimental results: squares are this work, circles are from ref 5 . Solid line is the fit of the terminal model to the data of this work with $r_{s}=0.48$ and $r_{m}=0.42$. Dashed line is the fit of the terminal model to the data of ref 5 with $r_{\mathrm{s}}$ $=0.53$ and $r_{\mathrm{m}}=0.49$.

Table II

Assignments to Peak Areas (Indicated by Chemical Shift) of the Aromatic Cl Region (Styrene-Centered Triads) in the ${ }^{13} \mathrm{C}$ NMR Spectra of Styrene-MMA Statistical Copolymers ${ }^{15}$

\begin{tabular}{cll}
\hline $\mathrm{X}^{\prime \prime}$ & \multicolumn{1}{c}{$\mathrm{Y}^{\prime \prime}$} & \multicolumn{1}{c}{$\mathrm{Z}^{\prime \prime}$} \\
$147.5-146.8 \mathrm{ppm}$ & \multicolumn{1}{c}{$146.8-145.15 \mathrm{ppm}$} & $145.15-143.5 \mathrm{ppm}$ \\
\hline$\left(1-\sigma_{\mathrm{sm}}\right)^{2} F_{\mathrm{msm}}$ & $\left(1-\sigma_{\mathrm{sg}}\right)^{2} F_{\mathrm{sss}}$ & $\left(1-\sigma_{\mathrm{ss}}\right) \sigma_{\mathrm{sm}} F_{\mathrm{ssm}}$ \\
& $2 \sigma_{\mathrm{ss}}\left(1-\sigma_{\mathrm{ss}}\right) F_{\mathrm{sss}}$ & $\sigma_{\mathrm{ss}} \sigma_{\mathrm{sm}} F_{\mathrm{ssm}}$ \\
& $\sigma_{\mathrm{ss}}{ }^{2} F_{\mathrm{sss}}$ & $\sigma_{\mathrm{sm}}{ }^{2} F_{\mathrm{msm}}$ \\
& $\left(1-\sigma_{\mathrm{ss}}\right)\left(1-\sigma_{\mathrm{sm}}\right) F_{\mathrm{ssm}}$ & \\
& $\sigma_{\mathrm{ss}}\left(1-\sigma_{\mathrm{sm}}\right) F_{\mathrm{ssm}}$ & \\
& $2 \sigma_{\mathrm{sm}}\left(1-\sigma_{\mathrm{sm}}\right) F_{\mathrm{msm}}$ &
\end{tabular}

Table III

Assignments to Peak Areas (Indicated by Chemical Shift) of the $\alpha$-Methyl Region (MMA-Centered Triads) in the ${ }^{13} \mathrm{C}$ NMR Spectra of Styrene-MMA Statistical Copolymers ${ }^{15}$

\begin{tabular}{|c|c|c|c|}
\hline$\stackrel{A}{A}$ & $\stackrel{\mathrm{B}}{21.2-19.8 \mathrm{ppm}}$ & $\stackrel{\mathrm{C}}{19.8-17.8 \mathrm{ppm}}$ & $\underset{17.8-16.5 \mathrm{ppm}}{\mathrm{D}}$ \\
\hline $\begin{array}{l}\sigma_{\mathrm{mm}} \cdot F_{\mathrm{mmm}} \\
\sigma_{\mathrm{mm}} \sigma_{\mathrm{sm}} F_{\mathrm{min}} \\
\sigma_{\mathrm{sm}}=F_{\mathrm{rm}}\end{array}$ & $\begin{array}{l}\sigma_{\mathrm{mm}}\left(1-\sigma_{\mathrm{sm}}\right) F_{\mathrm{mms}} \\
2 \sigma_{\mathrm{sm}}\left(1-\sigma_{\mathrm{sm}}\right) F_{\mathrm{sms}} \\
\left(1-\sigma_{\mathrm{sm}}\right)^{2} F_{\mathrm{sms}}{ }^{a}\end{array}$ & $\begin{array}{l}2 \sigma_{\mathrm{mm}}\left(1-\sigma_{\mathrm{mm}}\right) F_{\mathrm{mmm}} \\
\left(1-\sigma_{\mathrm{mm}}\right) \sigma_{\mathrm{sm}} F_{\mathrm{smm}} \\
\left(1-\sigma_{\mathrm{mm}}\right)\left(1-\sigma_{\mathrm{sm}}\right) F_{\mathrm{smm}} \\
\left(1-\sigma_{\mathrm{sm}}\right)^{2} F_{\mathrm{sms}}{ }^{\mathrm{s}}\end{array}$ & \\
\hline
\end{tabular}

a This peak shows resonance splitting and appears in both the $B$ and $\mathrm{C}$ peak areas.

The two regions of interest in the ${ }^{13} \mathrm{C}$ NMR spectra of the styrene-MMA copolymers are the aromatic $\mathrm{C} 1$ region on the styrene unit (chemical shift $(\delta)=148-143 \mathrm{ppm}$ ) and the $\alpha$-methyl region on the MMA unit ( $\delta=25-15$ ppm). These two regions show induced splitting due to the various tacticities and sequences of the styrenecentered triads and MMA-centered triads, respectively. In Figures 4 and 5 the ${ }^{13} \mathrm{C} \mathrm{NMR}$ spectra of these regions are displayed, showing the distinct peak areas. The assignments of these peak areas have been elucidated in the accompanying paper ${ }^{15}$ and for each of the sets of triad fractions are given in Tables II and III. For the styrenecentered triads there are three distinct peak areas, labeled $\mathrm{X}^{\prime \prime}(\delta=147.5-146.8 \mathrm{ppm}), \mathrm{Y}^{\prime \prime}(\delta=146.8-145.15 \mathrm{ppm})$, and $Z^{\prime \prime}(\delta=145.15-143.5 \mathrm{ppm})$. For the MMA-centered triads there are four distinct peak areas, labeled $\mathrm{A}(\delta=$ 23-21.2 ppm), B $(\delta=21.2-19.8 \mathrm{ppm}), \mathrm{C}(\delta=19.8-17.8$ ppm), and $D(\delta=17.8-16.5 \mathrm{ppm})$. The relative areas of the peaks for all copolymer compositions are given in Table IV. Note that in what follows for the MMA-centered triad peak areas $B$ and $C$ are considered as one, since the configuration, $\left(1-\sigma_{\mathrm{sm}}\right)^{2} F_{\mathrm{sm} g}$, is split between these peak areas. Therefore both the styrene-centered and MMAcentered triads are split into three effective peak areas. In the case of MMA-centered triads these are A, B + C, and D.

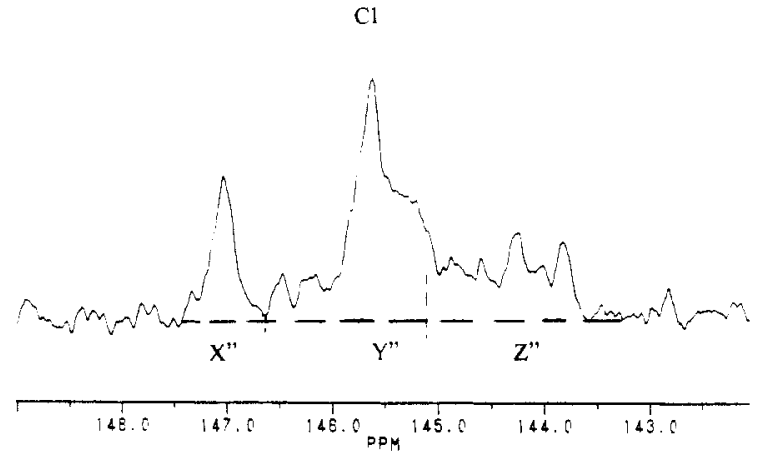

Figure 4. Expanded $100-\mathrm{MHz}^{13} \mathrm{C}$ NMR spectrum of styreneMMA copolymer (fraction of styrene in feed, $f_{\mathrm{s}}=0.377$ ) in $\mathrm{CDCl}_{3}$ at $298 \mathrm{~K}$, showing only the aromatic $\mathrm{C} 1$ region $(\delta=150-142 \mathrm{ppm})$ and three designated peak areas $\mathrm{X}^{\prime \prime}, \mathrm{Y}^{\prime \prime}$, and $\mathrm{Z}^{\prime \prime}$.

$$
\alpha-\mathrm{CH}_{1}
$$

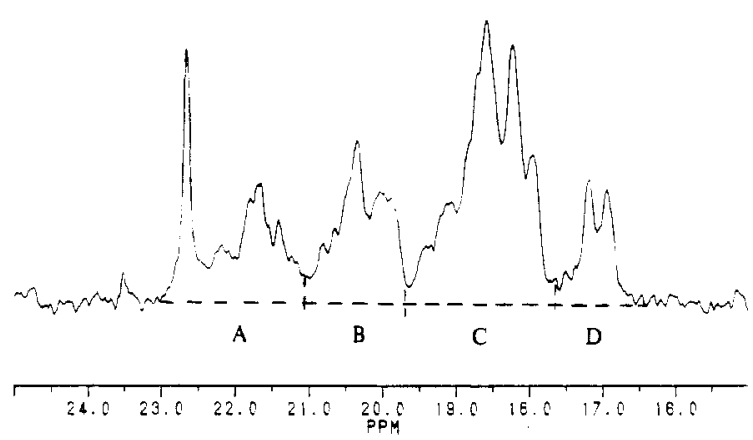

Figure 5. Expanded $100-\mathrm{MHz}^{13} \mathrm{C}$ NMR spectrum of styreneMMA copolymer (fraction of styrene in feed, $f_{\mathrm{s}}=0.377$ ) in $\mathrm{CDCl}_{3}$ at $298 \mathrm{~K}$, showing only the $\alpha$-methyl carbon region $(\delta=25-15$ $\mathrm{ppm}$ ) and four designated peak areas $\mathrm{A}, \mathrm{B}, \mathrm{C}$, and $\mathrm{D}$.

First, we show that the peak areas are predicted by the reactivity ratios found by a fit of the terminal model to the copolymer composition data in the previous part of this section $\left(r_{\mathrm{s}}=0.48 \pm 0.03\right.$ and $\left.r_{\mathrm{m}}=0.42 \pm 0.09\right)$. It is important to note that the styrene-centered triads are only determined by $r_{\mathrm{s}}$ and that the MMA-centered triads are only determined by $r_{\mathrm{m}}$. In Figure 6 it can be seen that the reactivity ratios calculated from polymer composition data predict the peak areas of the ${ }^{13} \mathrm{C}$ NMR spectra with spectacular success at all fractions of monomer. It is important to note that the assignments of the peak areas were elucidated by a technique entirely independent of any kinetic model for copolymerization and rely entirely upon NMR experimental data. Further, these assignments are different from those previously deduced elsewhere. ${ }^{15}$

For completeness we utilized the ${ }^{13} \mathrm{C}$ NMR data to predict reactivity ratios. From each set of data, i.e., styrene-centered and MMA-centered results, we find an independent reactivity ratio, $r_{B}$ and $r_{m}$, respectively. Utilizing the nonlinear least squares technique discussed above, we find $r_{\mathrm{s}}=0.51 \pm 0.05$ and $r_{\mathrm{m}}=0.52 \pm 0.02$. These values compare very well to those found from composition data: the standard deviations of the two sets of reactivity ratios overlap in each case. The fit of these parameters to the peak areas can be seen in Figure 7. A fit of the penultimate model to the ${ }^{13} \mathrm{C}$ NMR peak area data showed no statistical improvement in the fit.

Hence, the ${ }^{13} \mathrm{C}$ NMR data suggest that there are no penultimate unit effects on the triad fractions of the copolymers of styrene and MMA. This does not entirely disprove penultimate unit effects on polymer microstructure since it could be argued that penultimate unit effects may turn up in, say, pentad sequences. However, the fit of the terminal model presented above is much better evidence for the absence of penultimate unit effects on 

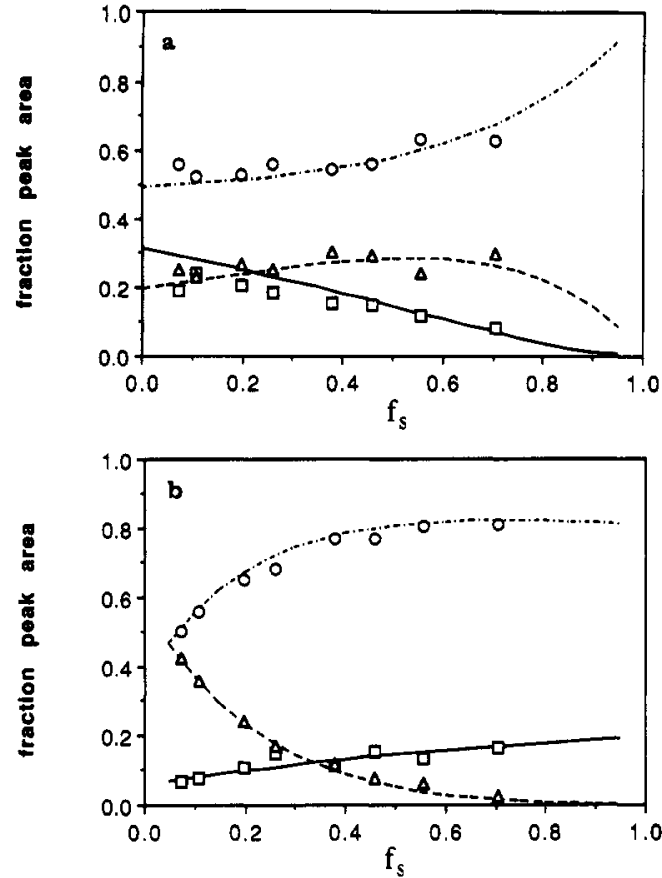

Figure 6. Fraction peak areas measured by ${ }^{13} \mathrm{C}$ NMR of styreneMMA copolymers polymerized at various fractions of styrene in the feed. Lines are predictions of terminal model utilizing reactivity ratios deduced from polymer composition data. (a) Styrene-centered peak areas: squares, peak area X"; circles, peak area $\mathrm{Y}^{\prime \prime}$; triangles, peak area $\mathrm{Z}^{\prime \prime}$. Lines calculated with $r_{\mathrm{s}}=0.48$. (b) MMA-centered peak areas: squares, peak area A; circles, peak areas $\mathrm{B}+\mathrm{C}$; triangles, peak area $\mathrm{D}$. Lines calculated with $r_{\mathrm{m}}=0.42$.

copolymer microstructure than simple polymer composition data.

Propagation Rate Coefficients in Copolymerization. The evidence that we present in this paper suggests that the terminal model is valid for both the polymer composition and the triad sequence distributions. The unusual feature of the copolymerization of styrene and methyl methacrylate is the now well-documented nonterminal model behavior ${ }^{5,6,11}$ of the mean propagation rate coefficient (see Figure 8). This behavior was explained by Fukuda et al. $.5,8$ by suggesting a restricted penultimate model. In this model the reactivity ratios, $r_{\mathrm{i}}$ and $r_{\mathrm{i}}{ }^{\prime}$ are set equal to each other; i.e.

$$
r_{i}=r_{i}^{\prime} \quad i=s \text { or } m
$$

From this equality it is found that the penultimate model reduces to the terminal model in the copolymer equation. Hence, although the mean propagation rate coefficient is influenced by penultimate unit effects, the composition and the triad sequence distributions are not.

The assumption that $r_{\mathrm{i}}=r_{\mathrm{i}}^{\prime}$ was initially made because of the good fit of the composition data to the terminal model. However, Moad et al. ${ }^{12}$ showed that this did not
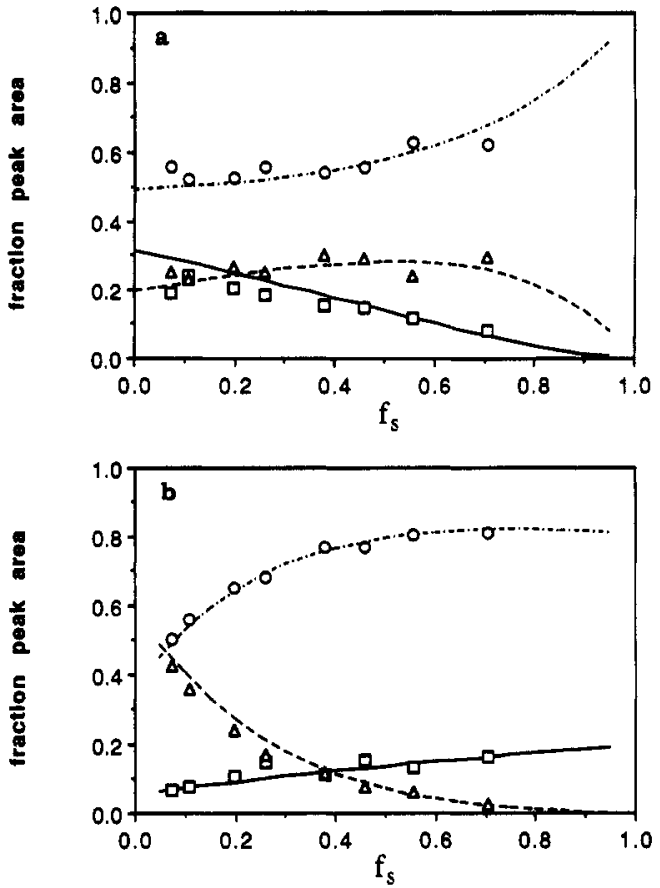

Figure 7. Fraction peak areas measured by ${ }^{13} \mathrm{C} \mathrm{NMR}$ of styreneMMA copolymers polymerized at various fractions of styrene in the feed. Lines are fits of the terminal model to the experimental points. (a) Styrene-centered peak areas: squares, peak area $\mathrm{X}^{\prime \prime}$; circles, peak area $Y^{\prime \prime}$; triangles, peak area $\mathrm{Z}^{\prime \prime}$. Lines calculated with fitted reactivity ratio, $r_{s}=0.51$. (b) MMA-centered peak areas: squares, peak area A; circles, peak areas B + C; triangles, peak area $\mathrm{D}$. Lines calculated with fitted reactivity ratio, $r_{\mathrm{m}}=$ 0.52 .

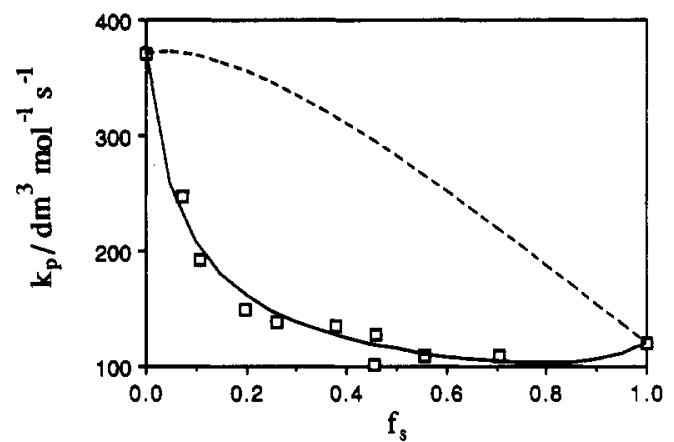

Figure 8. Plot of $k_{\mathrm{p}}$ versus $f_{\mathrm{s}}$ for styrene-MMA copolymerization at $40^{\circ} \mathrm{C}$. Experimental results (squares) from ref 5 . Solid line, predictions of restricted penultimate model with $s_{\mathrm{s}}=0.30$ and $s_{\mathrm{m}}=0.53$. Dashed line, prediction of terminal model with $r_{\mathrm{s}}=$ 0.52 and $r_{m}=0.46$.

necessarily mean that penultimate unit effects are not operating in regards to composition. Therefore, Fukuda et al. ${ }^{8}$ justified the use of eq 28 by considering a stabilization energy argument. There are problems with this justification, namely, that to find eq 28 Fukuda et al. made the following assumptions: ${ }^{8}$

Table IV

Summary of ${ }^{13} \mathrm{C}$ NMR Results Showing Fraction Peak Areas for Copolymers Produced at Various Fractions of Styrene in the Feed, $f_{s}{ }^{15}$

\begin{tabular}{|c|c|c|c|c|c|c|c|}
\hline \multirow[b]{2}{*}{$f_{8}$} & \multicolumn{4}{|c|}{ MMA-centered triads } & \multicolumn{3}{|c|}{ styrene-centered triads } \\
\hline & A & $\mathbf{B}$ & $\mathrm{C}$ & $\overline{\mathrm{D}}$ & $\mathbf{X}^{\prime \prime}$ & $\overline{Y^{\prime \prime}}$ & $\mathbf{Z}^{\prime \prime}$ \\
\hline 0.073 & 0.069 & 0.045 & 0.458 & 0.428 & 0.191 & 0.557 & 0.252 \\
\hline 0.109 & 0.079 & 0.066 & 0.494 & 0.361 & 0.243 & 0.524 & 0.232 \\
\hline 0.199 & 0.106 & 0.121 & 0.530 & 0.243 & 0.203 & 0.528 & 0.269 \\
\hline 0.262 & 0.148 & 0.153 & 0.528 & 0.171 & 0.187 & 0.561 & 0.252 \\
\hline 0.377 & 0.113 & 0.241 & 0.526 & 0.120 & 0.155 & 0.542 & 0.308 \\
\hline 0.458 & 0.154 & 0.255 & 0.516 & 0.075 & 0.148 & 0.558 & 0.293 \\
\hline 0.557 & 0.135 & 0.366 & 0.437 & 0.062 & 0.116 & 0.633 & 0.241 \\
\hline 0.705 & 0.166 & 0.478 & 0.330 & 0.025 & 0.080 & 0.624 & 0.296 \\
\hline
\end{tabular}


(1) Fukuda et al. assumed that the energy change for the addition of a monomer to a radical may be expressed as the algebraic sum of a normal energy change which would be observed in the absence of conjugation (or other effects) plus corrections due to the latter. ${ }^{19}$

$$
-\Delta H_{\mathrm{ijl}}=-\left(\Delta H_{\mathrm{p}}\right)_{0}+U_{\mathrm{jl}}-\left(U_{\mathrm{ij}}+U_{\mathrm{l}}\right)
$$

where $-\Delta H_{i j 1}$ is the energy change for the addition of monomer $\mathrm{L}$ to polymeric radical $\mathrm{IJ}^{\circ}$ (where $\mathrm{i}, \mathrm{j}, \mathrm{l}=\mathrm{s}$ or $\mathrm{m}$; $\mathrm{I}, \mathrm{J}, \mathrm{L}=\mathrm{S}$ or $\mathrm{M}),\left(\Delta H_{\mathrm{p}}\right)_{0}$ is the energy which would be observed in the absence of substituents, and $U_{\mathrm{jl}}, U_{\mathrm{ij}}$, and $U_{1}$ are the stabilization energies of the product radical, the initial radical, and the monomer, respectively.

(2) Fukuda et al. used the Polanyi rule for propagating free radicals. Hence the activation energy of propagation radical $\mathrm{IJ}^{\circ}$ with monomer $\mathrm{L}, E_{\mathrm{ijl}}$, can be written as ${ }^{19}$

$$
E_{\mathrm{ijl}}=\left(E_{\mathrm{ijl}}\right)_{0}-\alpha\left(-\Delta H_{\mathrm{ijl}}\right)
$$

where $\left(E_{\mathrm{ijl}}\right)_{0}$ and $\alpha$ are constants.

(3) Finally, Fukuda et al. neglected all constants and frequency factors and claimed that the penultimate propagation rate coefficients can be written as ${ }^{8}$

$$
k_{\mathrm{ij} 1} \approx \exp \left[\alpha\left(U_{\mathrm{j} 1}-U_{\mathrm{ij}}-U_{1}\right)\right]
$$

The use of eq 31 gives eq 28. Ignoring the not inconsiderable assumptions ${ }^{19}$ made in both the derivations and use of eqs 29-30 for polymeric radicals, we would like to concentrate on the assumptions made in the derivation of eq 31 . The use of eq 31 does not seem reasonable for the styrene-MMA system when it is realized that preexponential factors may be significantly different for various polymeric radicals. For example, the preexponential factors, $A$, for the homopolymerizations of the following systems have been experimentally determined: ${ }^{6}$ styrene $\left(A=1.99 \times 10^{7}\right)$, ethyl methacrylate $\left(A=1.5 \times 10^{6}\right)$, butyl methacrylate $\left(A=3.44 \times 10^{6}\right)$, MMA $\left(A=4.92 \times 10^{5}\right)$, and lauryl methacrylate $\left(A=2.93 \times 10^{5}\right)$. Note that the preexponential factors for the homopolymerization of styrene and MMA differ by approximately 2 orders of magnitude. In the copolymerization case there is no obvious a priori reason to assume that the preexponential factors for two radicals with differing penultimate units should be the same. In fact, this is a major assumption made by Fukuda et al. (without comment) and ensures the result of eq 28 .

Despite the above theoretical doubts, the restricted penultimate model $1^{5,8}$ does explain all the data and hence deserves further study, especially from a theoretical point of view. The question remains whether the fit of the restricted penultimate model is only a function of the number of adjustable parameters in the model. Without doubt, unambiguous experimental evidence of a penultimate unit effect must be observed before the restricted penultimate unit is adopted. In the styrene-MMA copolymerization system ESR may distinguish a penultimate unit effect, since the MMA propagating free radical has a well-characterized signal.

Monomer Concentrations in Copolymerization. The mean propagation rate coefficients in copolymerization are measured by at least two techniques, rotating sector ${ }^{5}$ and laser flash photolysis. 6 In both these techniques one major assumption is that the monomer concentration at the polymerization site is equal to that in the bulk phase. The bootstrap model ${ }^{7}$ proposes a local monomer ratio at the polymerization site that is different from the bulk monomer ratio. For the styrene-MMA polymerizing system it has been suggested that a bootstrap effect does in fact exist; ${ }^{9}$ however, this work utilized date that calculated triad fractions from ${ }^{1} \mathrm{H}$ NMR spectra-an approach that has been shown to be incorrect. ${ }^{14}$ We also note that if the ratio of monomers is affected by a bootstrap effect, then so may the total concentration of monomers. If this is the case, then the measurements of the mean propagation rate coefficients are subject to artifact.

In what follows we consider two manners by which local monomer concentrations and/or ratios at the polymerization site could arise. First, we look at bulk thermodynamics to determine the relationship, if any, between the monomer concentration at the polymerization site and the bulk monomer phase. Second, we consider that the proposed local monomer concentration at the polymerization site is a result of molecular-scale interactions between the monomers and polymer.

Bulk Monomer-Polymer Thermodynamics. In polymer kinetic studies a bulk solution containing low percentages of polymer and two monomers where there is no entanglement of the polymer chains has normally been considered as a homogeneous system but may in fact also be considered as a heterogeneous system containing globules of polymer surrounded by monomer. The dimensions of the globules will presumably be defined by the root-mean-square end-to-end distance of the polymer molecule in the particular monomer (solvent) mixture. The concentration of monomer in the globules may be lower than that in the bulk (monomer) phase. This is a situation analogous to latex particles swollen with monomer.

Maxwell et al. ${ }^{20}$ measured the concentration of two monomers in latex particle systems. They found for a variety of monomers, with a number of polymer latices, that in each case the ratio of the monomer concentrations was equal in the free monomer phase and the polymer (latex particle) phase. Maxwell et al. ${ }^{20}$ considered the thermodynamics of latex particle swelling by two monomers in terms of Flory-Huggins theory. By making reasonable assumptions, theory predicted that the ratio of two monomers in the bulk phase will be equal to that in the polymer-monomer phase. This result has been shown to be true for a variety of monomer and polymers in the latex particle situation. This fact would argue against a bootstrap effect that was caused by bulk thermodynamic effects. However, as will be argued later in this section, this does not discount the possibility of local monomer ratios.

In both the bulk and latex particle systems the concentrations of the two monomers in the polymer phase will always be less than in the pure monomer phase. However, simple calculations utilizing Flory-Huggins theory show that it is unlikely, for monomers like styrene and MMA and copolymers thereof, that the concentration of monomer in the polymer globule phase will be significantly less than that in the pure monomer phase. ${ }^{19}$ Even in latex particle systems where the degree of swelling of polymer by monomer is limited, not only by normal constraints but also by the latex particle surface tension, the monomer concentration typically attains $75 \%$ of its maximum value. ${ }^{20}$ Therefore it is very unlikely that the bulk monomer concentration in the polymer globule phase will cause a significant artifact in the measurement of mean propagation rate coefficients.

Monomer-Polymer Complezes. As alluded to above, the second manner by which the local monomer concentration at the polymerization site may differ from that in the bulk phase is by a "template" effect or "solventpolymer complex" effect. ${ }^{10}$ These concepts suggest that there are specific molecular interactions between the two 
monomers (solvents in the case of styrene and MMA) and polymer that cause the local monomer concentration and/ or ratio to be different at the polymerization site than in the free monomer phase. This type of effect has been observed in many copolymerization systems. ${ }^{10}$ It has also been noted that the apparent reactivity ratios of the styrene-MMA copolymerization system are dependent upon the solvent utilized during polymerization. These results can be interpreted by, inter alia, models based upon monomer-polymer complex effects.

For reasons discussed in the previous section, we do not consider that the total local monomer concentration could vary greatly from the bulk value. Hence, in what follows we assume that the deviation of the measured propagation rate coefficient from the predictions of the terminal model is caused by local monomer ratios that differ from the bulk monomer values. First we develop the models that follow from these propositions. We then fit these models to the data and see if the values of the fitted parameters are reasonable. In total, two different models are fitted to the data: one of these is a limit of the other.

In the following work an extension to the bootstrap model (eq 27) is developed to account for true monomer ratios, since these may vary with monomer ratio or polymer composition (which itself depends on the monomer ratio). The motivation behind this approach is that if, say, a monomer-polymer complex exists, then it is possible that the relationship between the bulk and true monomer ratios will be nonlinear with monomer ratio. There is no a priori reason to think that the bootstrap effect is constant for various monomer ratios. We describe this complex bootstrap effect by an extension to eq 27 :

$$
x_{\mathrm{t}}=\frac{\left[\mathrm{A}_{\mathrm{t}}\right]}{\left[\mathrm{B}_{\mathrm{t}}\right]}=K\left(\frac{[\mathrm{A}]}{[\mathrm{B}]}\right)^{2}
$$

where $K$ and $z$ are dummy variables. This versatile empirical form of the monomer ratio will hopefully be capable of fitting any real functional form. Note that if we set $z=1$, eq 32 reduces to eq 27 . In this case there is a single bootstrap parameter, $K$.

Now the mean propagation rate coefficients and polymer compositions measured by Fukuda et al..$^{5}$ are simultaneously fitted by the terminal model (eqs 2 and 4) with the further adjustable parameters that describe the monomer partitioning in the "complex" bootstrap model (eq 32). We designate the case where $z=1$ as the simple bootstrap terminal model (SBTM) and where $z \neq 1$ as the complex bootstrap terminal model (CBTM): this latter model has one extra adjustable parameter, $z$. We do not fit these models to the triad sequence distribution data measured in this work since we have already determined that these data are well fitted by the terminal model. Hence, any set of parameters in the terminal model that reproduce the polymer composition also reproduce the triad sequence distribution data.

It should be noted that the penultimate model has six adjustable parameters and that the restricted penultimate model described by Fukuda et al..$^{5}$ has four adjustable parameters. The two models utilized in this section have three (SBTM) and four (CBTM) adjustable parameters respectively. Given the agreement to data of the limited penultimate model, it is expected that both these models will provide adequate fits to experiment. It should be noted that a half-penultimate model ${ }^{21}$ in which only one of the radicals is allowed to have a penultimate unit effect also fits both the polymer composition and rate data. This model has only three adjustable parameters. However,

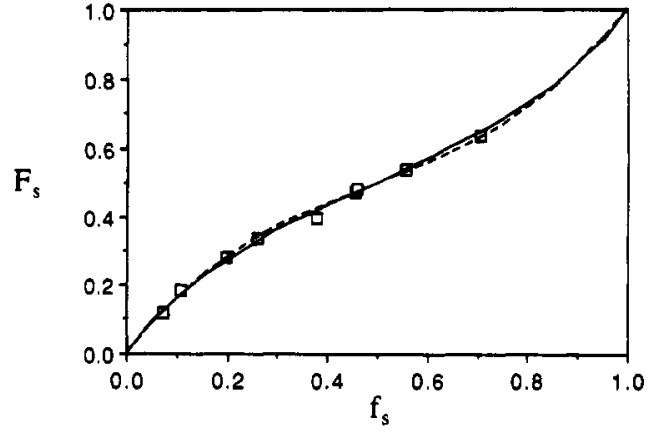

Figure 9. Fraction of styrene in copolymer versus fraction of styrene in feed. Experimental results (squares) from ref 5 . Solid line is the fit of the simple bootstrap terminal model (SBTM) with $r_{\mathrm{s}}=2.41, r_{\mathrm{m}}=0.11$, and $K=0.22$. Dashed line is the fit of the complex bootstrap terminal model (CBTM) with $r_{\mathrm{g}}=2.32$, $r_{\mathrm{m}}=0.05, K=0.15$, and $z=1.17$.

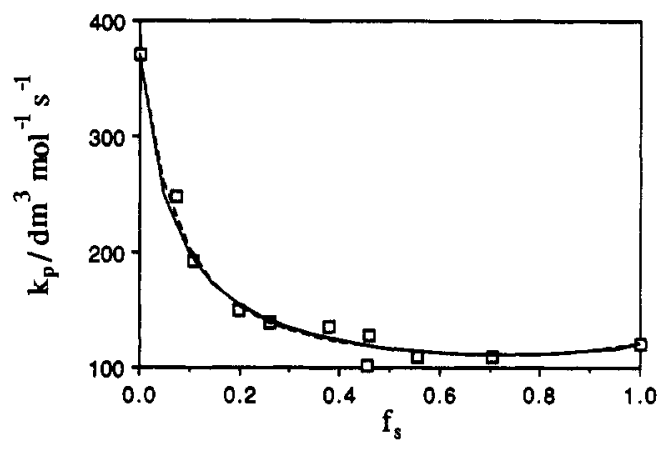

Figure 10. Plot of $k_{\mathrm{p}}$ versus $f_{\mathrm{s}}$ for styrene-MMA copolymerization at $40^{\circ} \mathrm{C}$. Experimental results (squares) from ref 5 . Solid line is the fit of the simple bootstrap terminal model (SBTM) with $r_{\mathrm{s}}=2.41, r_{\mathrm{m}}=0.11$, and $K=0.22$. Dashed line is the fit of the complex bootstrap terminal model (CBTM) with $r_{\mathrm{s}}=2.32$, $r_{\mathrm{m}}=0.05, K=0.15$, and $z=1.17$.

we cannot see any theoretical justification for the use of this model.

Utilizing the same nonlinear least squares fitting package as described in an earlier section, ${ }^{17}$ the fit to the polymer composition and mean $k_{\mathrm{p}}$ data of Fukuda et al. ${ }^{5}$ by both the simple and complex bootstrap terminal models gives the following parameters: (1) SBTM, $r_{s}=2.41 \pm 0.12, r_{m}$ $=0.11 \pm 0.03$, and $K=0.22 \pm 0.05 ;(2)$ CBTM, $r_{\mathrm{s}}=2.32$ $\pm 0.13, r_{\mathrm{m}}=0.05 \pm 0.04, K=0.15 \pm 0.07$, and $z=1.17 \pm$ 0.16 . In both cases a more than adequate fit to the data is described (Figures 9 and 10). Note that the use of four parameters in the CBTM when three parameters provide an adequate fit in the SBTM indicates a possible lack of uniqueness in some parameters. In fact, the functional relationship model ${ }^{17}$ indicates that in the SBTM the parameters $r_{\mathrm{m}}$ and $K$ are highly correlated (i.e., have a large joint confidence region), and in the CBTM the parameters $K$ and $z$ are correlated to $r_{m}$. Statistically, the CBTM shows no improvement in the fit to the data over the SBTM.

The values of the reactivity ratios suggest that the "apparent" reactivity ratios are very different from the real reactivity ratios predicted by this model. Deviations of this magnitude obviously need independent experimental correlation. More disturbing is the deviation of the bulk and true monomer concentrations. If we concentrate upon the results of the SBTM, we see that the ratio of the concentrations of styrene and MMA is 0.22 times that in the bulk (or feed). That is, complex formation between the copolymer and MMA is favored over that between copolymer and styrene. If a monomer-copolymer complex does exist, then it is possible that this complex depends upon the copolymer structure. That is, say, a 
specific interaction between MMA in the copolymer and the MMA monomer could be favored at high MMA content in the copolymer. If this is the case, then the value of $z$ in the CBTM should be far from unity. In the CBTM the fitted value of $z$ is slightly larger than unity, suggesting that there may be only a slight polymer composition effect on the bootstrap effect.

There is some experimental basis for considering that a monomer-polymer complex effect may occur in copolymerizations of styrene and MMA, namely, the (sometimes large) solvent effect upon the reactivity ratios. ${ }^{10}$ However, apart from this fact and the ability of the above models to fit data, there is little evidence for the veracity of these bootstrap models. The same is also true of the penultimate and restricted penultimate models, which have no theoretical nor experimental grounding. One possible direct experimental test for the presence of monomer-polymer complexes would be a fluorescence study in which fluorescence probes built into the polymer are quenched by two monomers at different rates. Varying the monomer ratio in time-resolved fluorescence studies may provide some direct evidence of a local monomer ratio around the polymer chain.

\section{Conclusions}

Triad sequence distributions for the model copolymerization system styrene and MMA have never been measured before since ${ }^{1} \mathrm{H}$ NMR spectra must be interpreted in terms of pentads ${ }^{14}$ and all previous ${ }^{13} \mathrm{C}$ NMR spectra have been interpreted with erroneous peak assignments. ${ }^{15}$ The triad sequence distribution of copolymers of styrene and MMA polymerized with differing monomer ratios in the bulk was well fitted by the terminal model. This does not entirely disprove penultimate unit effects on polymer microstructure but is much better evidence for the absence of them than simple polymer composition data.

All current copolymerization data on the styrene-MMA system, including the triad sequence distribution data measured in this work, can be explained by the restricted penultimate model. ${ }^{5}$ However, this model has no good theoretical basis and is also difficult to test by direct experiment. The model may work simply because it contains sufficient adjustable parameters. Despite these doubts the model does explain all the data, and hence deserves further study, especially from a theoretical point of view. Further experimental evidence of a penultimate unit effect must be observed before the restricted penultimate unit is adopted. In the styrene-MMA copolymerization system ESR may distinguish a penultimate unit effect.

New models which incorporate the bootstrap model ${ }^{7}$ or monomer-solvent complex model ${ }^{10}$ into the terminal model for polymerization can also explain all the existing data on the styrene-MMA polymerizing system. These models presume that the local monomer ratio at the polymerization site is changed from that in the bulk monomer phase. There is some evidence for a solventpolymer complex effect for this system, namely, the variation in measured reactivity ratios with solvent. ${ }^{10}$ If a complex between copolymer and monomers does in fact exist, then this should be detectable by experiment. For example, quenching by monomers of a fluorescence probe incorporated into copolymer could provide evidence for the presence or absence of a monomer-polymer complex.

In conclusion, the triad sequence distributions measured in this work for the styrene-MMA system allow us to state with more authority that there is no penultimate unit effect on polymer chain structure. The presence of a nonterminal unit effect on the rate of polymerization (the mean propagation rate coefficient) can be explained by invoking a number of models. Many of these models have been previously described, and in this work new interpretations of previously existing models are developed: the monomerpolymer complex and bootstrap models are adapted to account for kinetic effects. Alternatively, new models can also be formulated that explain all extant copolymerization data; e.g., if the rates of propagation were dependent upon polymer tacticity, then a model similar in algebraic form to the penultimate model could be developed, but with different interpretation of the various reactivity ratios. The work of this paper suggests that a successful (and physically reasonable) model must have at least three adjustable parameters. However, there is no double that model discrimination can only proceed if further independent experiments designed to test particular models are performed.

Acknowledgment. The authors would like to thank Ken O'Driscoll, Jenci Kurja, Bert Klumpermans, Bob Gilbert, Harold Schoonbrood, Nico Linssen, and Alex van Herk for their valuable discussions relating to this work.

\section{References and Notes}

(1) Mayo, F. R.; Lewis, F. M. J. Am. Chem. Soc. 1944, 66, 1954.

(2) Bandrup, J.; Immergut, E. H. Polymer Handbook, 3rd ed.; Wiley: New York, 1989.

(3) Fukuda, T.; Ma, Y.-D.; Inagaki, H. Polym. J. 1982, 14, 705.

(4) Hill, D. J.T.; O'Donnell, J.H.; O'Sullivan, P.W. Macromolecules $1982,15,960$.

(5) Fukuda, T.; Ma, Y.-D.; Inagaki, H. Macromolecules 1985, 18, 17.

(6) Davis, T. P.; O'Driscoll, K. F.; Piton, M. C.; Winnik, M. A. Macromolecules 1989, 22, 2785.

(7) Harwood, H. J. Makromol. Chem., Macromol. Symp. 1987, 10/ $11,331$.

(8) Fukuda, T.; Ma, Y.-D.; Inagaki, H. Makromol. Chem., Rapid Commun. 1987, 8, 495.

(9) Davis, T. P. Polym. Commun. 1990, 31, 442.

(10) Barton, J.; Borsig, E. Complexes in Free Radical Chemistry; Elsevier: Amsterdam, 1988.

(11) O'Driscoll, K. F.; Davis, T. P. J. Polym. Sci. 1989, $27,417$.

(12) Moad, G.; Solomon, D. H.; Spurling, T. H.; Stone, R. A. Macromolecules 1989, 22, 1145 .

(13) Tanaka, H.; Sasai, K.; Sato, T.; Ota, T. Macromolecules 1988, $21,3536$.

(14) Aerdts, A. M.; de Haan, J. W.; German, A. L.; v. d. Velden, G. P. M. Macromolecules 1991, 24, 1473.

(15) Aerdts, A. M.; de Haan, J. W.; German, A. L. Macromolecules, accompanying paper, in this issue.

(16) Koenig, J. L. Chemical Microstructure of Polymer Chains; Wiley: New York, 1980.

(17) Hillegers, L. T. M. E. The Estimation of Parameters in Functional Relationship Models. Ph.D. Dissertation, Eindhoven University of Technology, The Netherlands, 1986.

(18) O'Driscoll, K. F.; Reilly, P. M. Macromol. Symp. 1987, 10/11, 355.

(19) Flory, P.J.Principles of Polymer Chemistry; Cornell University Press: Ithaca, NY, 1953.

(20) Maxwell, I. A.; Kurja, J.; van Doremaele, G. H. J.; German, A. L. Makromol. Chem. 1992, 193, 2065.

(21) Maxwell, I. A.; Napper, D. H.; Gilbert, R. G., to be published. Louis, P. La Polymerisation Radicalaire Libre du Styrene en Milieux Heterogenes. Ph.D. Dissertation, University of Liège, Belgium, 1990 